\title{
Pauvreté et développement dans un monde globalisé
}

L'aide au développement aide-t-elle le développement?

\section{François Bourguignon}

\section{OpenEdition}

\section{Journals}

Édition électronique

URL : https://journals.openedition.org/lettre-cdf/1940

DOI : $10.4000 /$ lettre-cdf. 1940

ISSN : 2109-9219

Traduction(s) :

Poverty and Development in a Globalized World: - URL : https://journals.openedition.org/lettre-cdf/ 2157 [en]

\section{Éditeur}

Collège de France

\section{Édition imprimée}

Date de publication : 1 mars 2015

Pagination : 9

ISSN : 1628-2329

\section{Référence électronique}

François Bourguignon, «Pauvreté et développement dans un monde globalisé », La lettre du Collège de France [En ligne], 39 | mars 2015, mis en ligne le 01 août 2015, consulté le 17 août 2022. URL : http:// journals.openedition.org/lettre-cdf/1940 ; DOI : https://doi.org/10.4000/lettre-cdf.1940 


\section{L'aide au développement aide-t-elle le développement ?}

\begin{abstract}
Dès la décolonisation, la coopération internationale a été considérée comme un instrument majeur pour une communauté soucieuse, pour des raisons humanitaires ou géopolitiques, de ne pas laisser une grande partie de la population mondiale dans le dénuement absolu.
\end{abstract}

Mais dans l'état actuel des connaissances sur l'efficacité de l'aide, les bailleurs de fonds sont confrontés à un dilemme. Ils font face à des bénéficiaires potentiels qui diffèrent selon leurs besoins, et selon leur efficacité à effectivement faire parvenir l'aide qu'ils reçoivent aux plus démunis. Ils doivent donc arbitrer entre des pays dont la gouvernance est meilleure mais la pauvreté moins aiguë qu'ailleurs, et des pays plus pauvres dont la gouvernance est souvent plus laxiste. Bien sûr, cet arbitrage n'aurait pas lieu d'être si les bailleurs de fonds pouvaient exercer un contrôle sur l'utilisation de l'aide. En pratique, cependant, ce contrôle est limité sauf dans des cas exceptionnels, comme lors de crises économiques ou humanitaires. Cette sélectivité de l'aide par caractéristiques des pays bénéficiaires est très directement observable et elle a des conséquences importantes.

Ainsi, l'Association internationale du développement, bras de la Banque mondiale responsable de la gestion des fonds d'aide que lui confient les pays donateurs, alloue ses ressources aux pays à bas-revenus sur la base d'une formule mathématique qui dépend de trois termes : un indice décrivant la qualité des institutions et des politiques mises en œuvre, le revenu par habitant, et la population des pays bénéficiaires. Le premier de ces facteurs, qui résume ce que l'on peut anticiper de la qualité de la gouvernance d'un pays, joue cependant un rôle démesuré.

Comme les agences nationales d'aide au développement dans les pays riches ont tendance à suivre la même stratégie, la conséquence en est que sont apparus ces dernières années de véritables « chouchous » mais aussi des « orphelins » de l'aide, selon que la gouvernance d'un pays est jugée plus ou moins satisfaisante. Par ailleurs, s'agissant de l'utilisation de l'aide, une nette évolution a eu lieu en faveur des secteurs dits " sociaux » - santé, éducation et transferts sociaux - au détriment de l'infrastructure et des travaux publics, où le détournement de fonds est réputé plus aisé.
Une telle stratégie d'allocation de l'aide est contre-productive. Les pays qui jouissent d'une meilleure gouvernance croissent souvent plus vite que les autres. L'aide s'oriente donc vers des populations où l'espérance d'une amélioration des conditions de vie est plus élevée et néglige des populations pauvres, mal gouvernées par des élites peu scrupuleuses et dont la pauvreté risque au contraire d'augmenter. Ces pays que la communauté des bailleurs de fonds qualifie de « fragiles " se trouvent donc plus ou moins laissés pour compte, et il est illusoire de penser que cette sanction touche uniquement les classes dirigeantes.

Un contrôle plus direct dans ces pays « fragiles » est peut-être la seule façon d'améliorer l'efficacité de l'aide au développement. Après tout, c'est la pratique de la Chine, dont une partie de l'aide au développement africain est livrée sous la forme d'infrastructures clés-en-main construites par des entreprises chinoises, court-circuitant ainsi le gouvernement et les entreprises locales. De fait, cette pratique est aujourd'hui interdite pour les bailleurs occidentaux au sein du Comité d'aide au développement de I'OCDE. Au reste, déresponsabiliser les élites dirigeantes ne paraît pas la meilleure manière de les inciter à gérer leurs économies de façon efficace et transparente.

Le débat sur la coopération internationale dans le domaine du développement semble se concentrer sur la question de l'aide. Mais l'analyse montre que les contraintes extérieures qui s'exercent sur les pays les plus pauvres ne peuvent être toutes levées par les seuls flux d'aide. Qu'il s'agisse des échanges commerciaux, des politiques migratoires, de l'environnement ou du transfert de connaissance, les politiques des pays riches et, de plus en plus, des pays émergents, sont susceptibles d'accélérer mais aussi dans certains cas de brider la croissance des pays moins développés. Plusieurs pays avancés particulièrement attentifs aux questions de développement cherchent aujourd'hui à introduire une cohérence explicite dans l'ensemble de leurs politiques qui peuvent affecter directement ou indirectement le monde en développement.

Extraits de la leçon inaugurale du 3 avril 2014

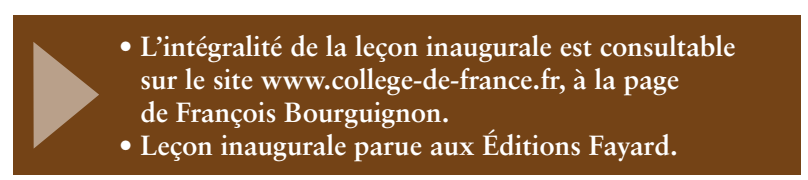

